\title{
LncRNA MIAT sponges miR-149-5p to inhibit efferocytosis in advanced atherosclerosis through CD47 upregulation
}

Zi-ming Ye ${ }^{1,2}$, Shuai Yang ${ }^{1}$, Yuan-peng Xia ${ }^{1}$, Rui-ting Hu${ }^{2}$, Shengcai Chen ${ }^{1}$, Bo-wei Li', Shao-li Chen ${ }^{1}$, Xue-ying Luo ${ }^{1}$, Ling Mao', Yanan Li ${ }^{1}$, Huijuan Jin ${ }^{1}$, Chao $\mathrm{Qin}^{2}$ and Bo Hu${ }^{1}$

\begin{abstract}
Atherosclerotic cardio-cerebrovascular disease and death remain the leading cause of morbidity and mortality worldwide. Defective efferocytosis, the clearance of apoptotic cells by macrophages, is thought to lead to increased inflammation and necrotic core formation in atherosclerotic lesions. However, very little is known about the role of long noncoding RNA (InCRNA) during this process. Here we show that IncRNA myocardial infarction associated transcript (MIAT) was markedly elevated in the serum of patients with symptoms of vulnerable atherosclerotic plaque and the macrophages of necrotic cores in an advanced atherosclerosis mouse model. MIAT knockdown attenuated atherosclerosis progression, reduced necrotic core size, and increased plaque stability in vivo. Furthermore, MIAT knockdown promoted clearance of apoptotic cells by macrophages in vivo and in vitro. Mechanistic studies revealed that MIAT acted as a micro RNA (miRNA) sponge to positively modulate the expression of anti-phagocytic molecule CD47 through sponging miR-149-5p. Together, these findings identified a macrophage MIAT/miR-149-5p /CD47 pathway as a key factor in the development of necrotic atherosclerotic plaques.
\end{abstract}

\section{Introduction}

Atherosclerotic plaque vulnerability has been identified as the major cause of the atherosclerotic cerebrovascular disease $^{1,2}$. While the majority of atherosclerotic plaques remain clinically silent, some instable plaques may suddenly rupture after decades of indolent progression and cause life-threating acute ischemic events ${ }^{3,4}$. Therefore, the ability to identify and stabilize vulnerable plaques would be of great value in patients at high risk of lesion rupture. Long noncoding RNAs (lncRNAs) belong to a class of non-protein-coding RNAs longer than 200 nucleotides involved in the epigenetic regulation by

\footnotetext{
Correspondence: Chao Qin (mdqc6639@126.com) or Bo Hu (hubo@mail.hust. edu.cn)

${ }^{1}$ Department of Neurology, Union Hospital, Tongji Medical College, Huazhong University of Science and Technology, 430022 Wuhan, China

${ }^{2}$ Department of Neurology, The First Affiliated Hospital, Guangxi Medical University, Nanning 530021 Guangxi, China

These authors contributed equally: Zi-ming Ye, Shuai Yang, Yuan-peng Xia Edited by A. Stephanou
}

modulating gene expression ${ }^{5}$. There is growing evidence implicating some atherosclerosis-related lncRNAs in plasma lipid homeostasis, such as cholesterol absorption, uptake of modified lipoproteins, and reverse cholesterol transport, thereby affecting the progression of atherosclerosis $^{6,7}$. Moreover, recent studies have shown that lncRNAs exist in the circulation and can serve as independent biomarkers reflecting the local disease process in cardiovascular disease ${ }^{8,9}$. Further investigation into the roles of lncRNAs in atherosclerotic plaque progression and vulnerability, as well as the exact mechanisms involved are needed.

Myocardial infarction associated transcript (MIAT), also termed as Gomafu in humans or Rncr2 in mice, is a highly conserved mammalian $\operatorname{lncRNA}{ }^{10,11}$. Many researchers have conducted studies and found that MIAT is involved in various physiological and pathological processes, including neuron development, formation of nuclear bodies, microvascular dysfunction, and 
myocardial infarction ${ }^{11-13}$. Recent reports based on the quantitative polymerase chain reaction (PCR) have indicated that MIAT is highly expressed in human carotid plaques and might be a potential diagnostic indicator in ischemic stroke $\mathrm{e}^{\mathrm{14}, 15}$. These results suggest that MIAT may have potential roles in atherosclerotic cerebrovascular disease. Therefore, in the current study we aimed to determine whether MIAT was a critical regulator of plaque vulnerability and to define the underlying molecular mechanisms.

In atherosclerosis, the uptake of modified lipoproteins by macrophages exceeds cholesterol efflux, leading to the deposition of cholesterol esters and the subsequent formation of foam cells ${ }^{16,17}$. Apoptosis and the secondary necrosis of foam cells are thought to be major causes of necrotic core development and contribute toward the formation of vulnerable plaques ${ }^{18,19}$. Under physiological condition, these apoptotic cells are rapidly cleared by macrophages and other phagocytes through a process known as efferocytosis ${ }^{20,21}$. Recent results have revealed that efferocytosis is defective in advanced plaques of human and animals, which may explain why necrosis cells constantly accumulate in the necrotic core and aggravate the inflammatory response ${ }^{18,22,23}$. Efferocytosis is mediated by macrophages recognizing phagocytic 'eat me' signals on the apoptotic cells and can be counterbalanced by anti-phagocytic 'don't eat me' signal such as the CD47 molecule ${ }^{20,24}$. Among the antiphagocytic signal molecules, CD47 has been identified as a novel therapeutic target for treating atherosclerosis by promoting efferocytosis $^{24}$. However, previous studies reported that antiCD47 antibody therapy contributed to splenic erythrophagocytosis and compensatory reticulocytosis ${ }^{25,26}$. In addition, it was found that the anti-CD47 antibodies are not curative alone, possibly due to the molecular weight of antibodies being so large and the limited tissue penetration ${ }^{27}$. Therefore, further studies are warranted to define additional measures to target the regulation of CD47 expression.

In the current study, we found that MIAT was significantly upregulated in serum of patients with symptoms of atherosclerotic vulnerable plaque and was consistently increased in serum and macrophages of necrotic cores in an advanced atherosclerosis mouse model. Subsequent knockdown of MIAT in vivo demonstrated that silencing of MIAT significantly enhanced phagocytic clearance and reduced atherosclerosis plaque progression and instability. Mechanistically, MIAT acted as a micro RNA (miRNA) sponge to positively modulate the expression of CD47 through sponging miR-149-5p. Therefore, our study provides new insights into the molecular function of the MIAT/miR-149-5p/CD47 signaling pathway in the pathogenesis of plaque vulnerability and highlights the potential of MIAT as a new therapeutic target for atherosclerosis.

\section{Results}

MIAT was upregulated in symptomatic human atherosclerotic disease and in advanced mouse atherosclerosis

Disease-associated lncRNAs can often be detected in the circulation and may biologically influence the local disease process ${ }^{7,8}$. We first assessed whether the expression of MIAT was altered in atherosclerotic disease using quantitative reverse transcription polymerase chain reaction (qRT-PCR) analysis of serum RNA extracted from the control group and atherosclerotic patients who had suffered either a transient ischemic attack or stroke (symptomatic) or were asymptomatic. In addition, to determine if the plaques in the atherosclerotic patients were stable or vulnerable, we used carotid magnetic resonance imaging (CMIR) to detect the subjects, CMIR is capable of accurately characterizing plaque morphology, composition, and surface condition and has been extensively validated by histology (Fig. 1a, b). We found that the serum of symptomatic subjects had higher levels of MIAT compared with those of asymptomatic patients and that serum MIAT levels were also increased in asymptomatic patients compared to those in the control group (Fig. 1c). The baseline and clinical characteristics of the study populations are shown in Supplementary Table 1. Comparison of some parameters including age, sex, and lipid profiles among the three groups showed no statistically significant differences. To further investigate the correlation of MIAT and atherosclerotic plaque progression, we detected its expression in $\mathrm{ApoE}^{-/-}$mice fed a high-fat diet (HFD) for 8 weeks, which induced relatively early atherosclerotic lesions, and in mice fed HFD for 16 weeks, which resulted in the development of advanced plaques. As expected, the expression levels of MIAT in the serum in HFD-fed $\mathrm{ApoE}^{-1-}$ mice were $\approx 1.83$-fold and $\approx 2.40$-fold higher, respectively, than that in normal chow diet (NCD)-fed $\mathrm{ApoE}^{-1-}$ mice at 8 and 16 weeks (Fig. 1d). In addition, the expression levels of MIAT in the aorta in HFD-fed $\mathrm{ApoE}^{-1-}$ mice were $\approx 2.56$-fold and $\approx 2.83$-fold higher, respectively, than that in NCD-fed ApoE ${ }^{-1-}$ mice at 8 and 16 weeks (Fig. 1e). These results suggested that MIAT may be involved in the progression and instability of atherosclerotic plaques.

\section{MIAT was upregulated in macrophages of advanced atherosclerotic lesions and was induced in vitro by ox-LDL}

To better understand the location and potential role of MIAT in advanced atherosclerosis, we performed RNA fluorescent in situ hybridization (RNA-FISH) on the advanced plaques. We found that the majority of the lesion macrophages expressed MIAT based on combined immunostaining for MIAT and Mac-3 (Fig. 2a). Additionally, a few smooth muscle cells (SMCs) also expressed MIAT (Supplementary Fig. 1). Furthermore, Raw264.7 

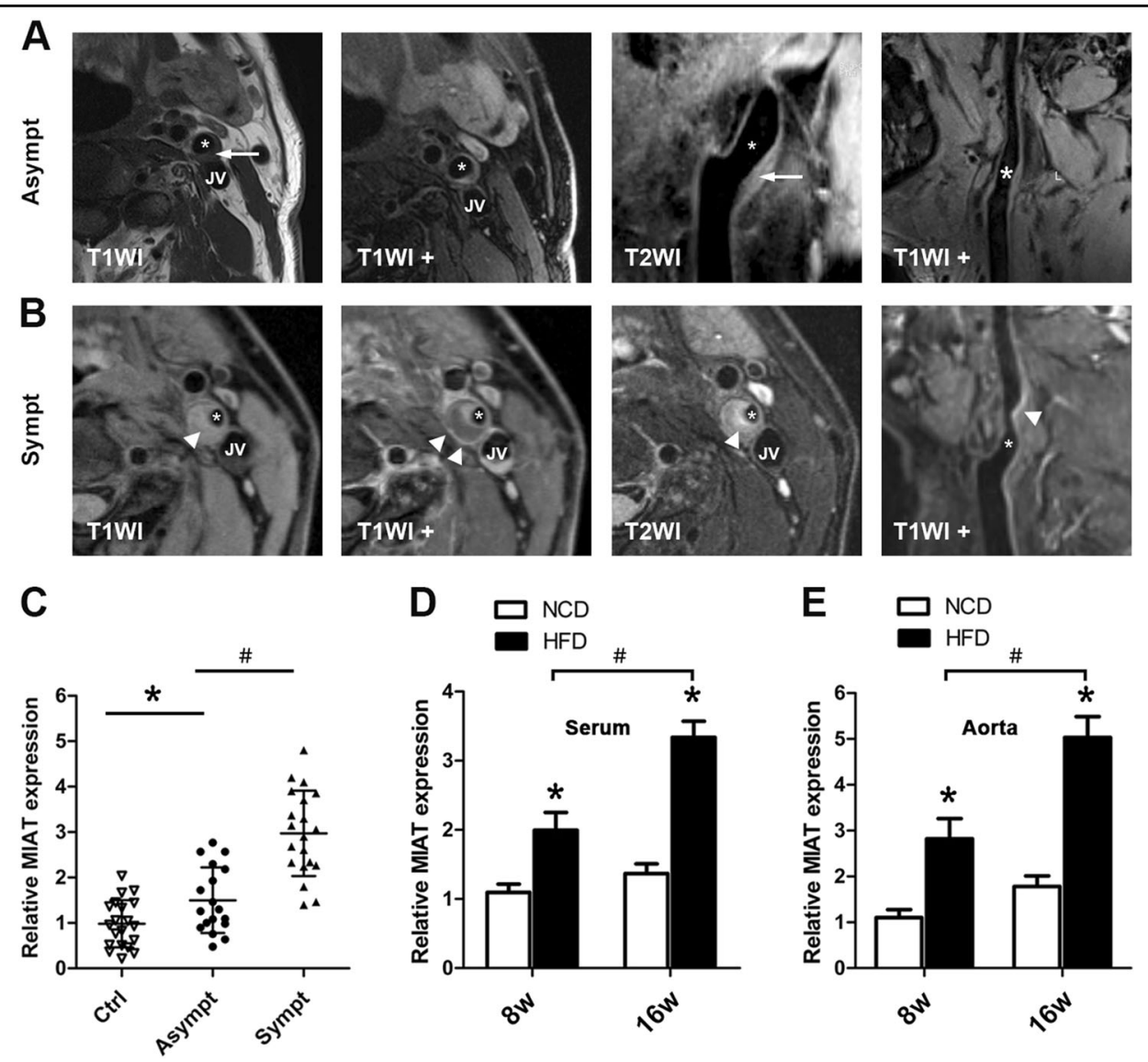

Fig. 1 Expression of MIAT increased in symptomatic human atherosclerotic disease and in advanced mouse atherosclerosis. a Example of type III lesion in the internal carotid artery (extracellular lipid pool was detected by histology). On multicontrast-weighted MR images, iso-intensity on T1WI and slightly higher signal on T2WI (arrow), no enhancement in T1WI + . The asterisks (*) indicates lumen. JV indicates jugular vein. $\mathbf{b}$ Example of type IV-V lesion in the internal carotid artery (lipid-rich necrotic core was detected by histology). On multicontrast-weighted MR images, lipid-rich necrotic core (arrowheads) had slightly higher signal on both T1WI and T2WI and had enhancement in T1WI + (arrowheads). Lumen is severely stenosed. The asterisks $\left(^{*}\right)$ indicates lumen. c Quantitative reverse transcriptase-polymerase chain reaction (qRT-PCR) analysis of expression of MIAT in the serum from control (Ctrl, $n=20$ ), asymptomatic (Asympt, $n=18$ ) or symptomatic (Sympt, $n=20$ ) subjects with recent $\mathrm{TIA} / \mathrm{stroke}\left({ }^{*} P<0.05\right.$, Asympt patients vs. Ctrl, ${ }^{\#} P<0.05$, Sympt patients vs. Asympt patients). $\mathbf{d}$, e Analysis of MIAT expression in the serum and aorta of atherosclerotic lesions of HFD-Fed ApoE ${ }^{-1-}$ mice and NCD-fed ApoE mice ${ }^{-/-}$at 8 and 16 weeks by qRT-PCR ( ${ }^{*} P<0.05$, HFD-fed ApoE ${ }^{-1-}$ mice vs. NCD-fed ApoE $^{-/-}$ mice, ${ }^{\#} P<0.05$, HFD-fed ApoE ${ }^{-1-}$ mice at 16 week vs. HFD-fed ApoE $^{-1-}$ mice at 8 week, $n=6 /$ group)

cells were stimulated with oxidized low-density lipoprotein (ox-LDL) or native LDL. Expression levels of MIAT were significantly increased after ox-LDL treatment in dose-dependent and time-dependent manners (Fig. 2b, c). We also determined by in vitro RNA-FISH that MIAT was localized predominantly in the cytoplasm and not in the nucleus and that MIAT expression markedly increased after ox-LDL treatment (Fig. 2d, e).

\section{Knockdown of MIAT attenuated atherosclerosis progression in $\mathrm{ApoE}^{-/-}$mice}

To explore the function and therapeutic potential of MIAT in vivo, seven-week-old $\mathrm{ApoE}^{-/-}$mice were fed the HFD for 4 weeks and then treated with PBS, viral scrambled small hairpin RNA (Scr shRNA), or MIAT shRNA and continued to be fed the HFD for 12 weeks
(Fig. 3a). First, we determined the efficacy of MIAT inhibition by measuring the expression of MIAT in the serum and aorta after 12 weeks of treatment. Expression levels of MIAT detected using qRT-PCR were significantly decreased in the MIAT shRNA-treated group compared with that in the Scr shRNA-treated group or PBS-treated group (Fig. 3b, c). After 12 weeks of feeding the animals with the HFD, there were no differences in body weight or levels of total cholesterol (TC), triglycerides (TG), low-density lipoproteins (LDL), or highdensity lipoproteins (HDL) among the MIAT shRNAtreated group, the scrambled shRNA-treated group, or PBS-treated group (Supplementary Figure 2). Quantification of the atherosclerotic lesions by Oil-red $\mathrm{O}$ staining after 12 weeks of HFD showed no significant differences between the Scr shRNA-treated group and 


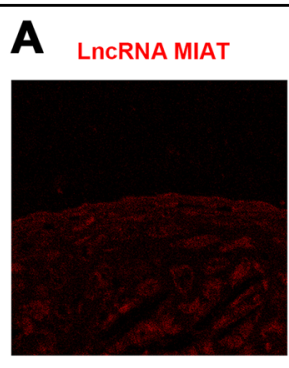

D
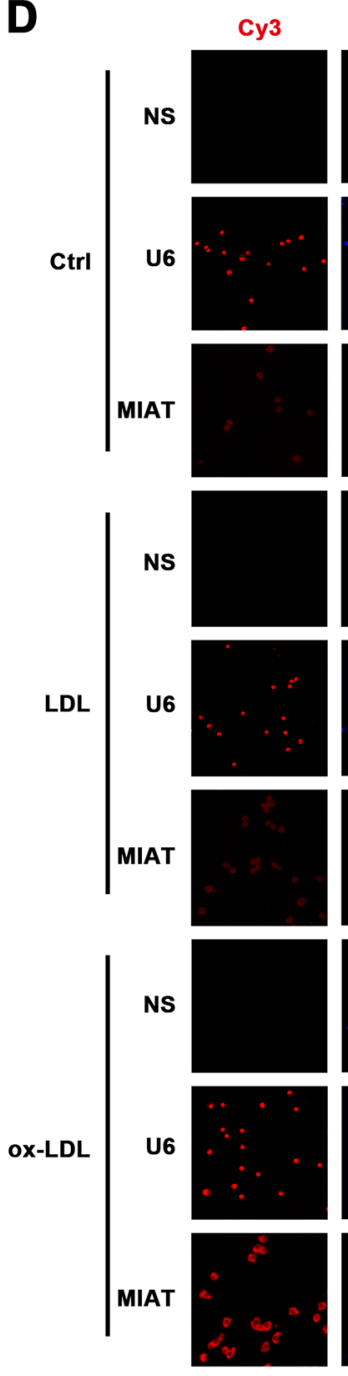
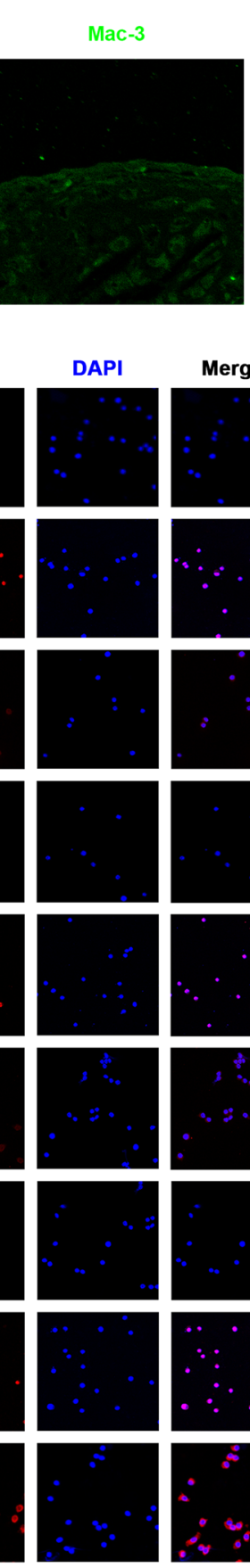

Merge
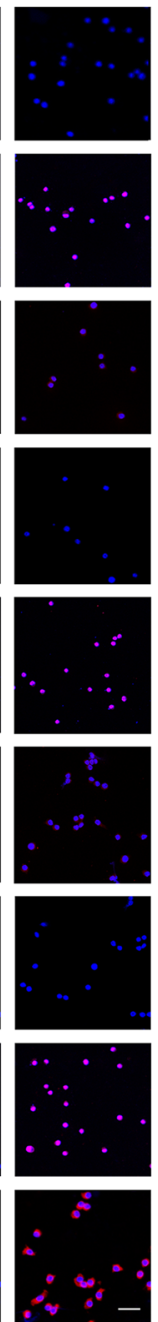

DAPI
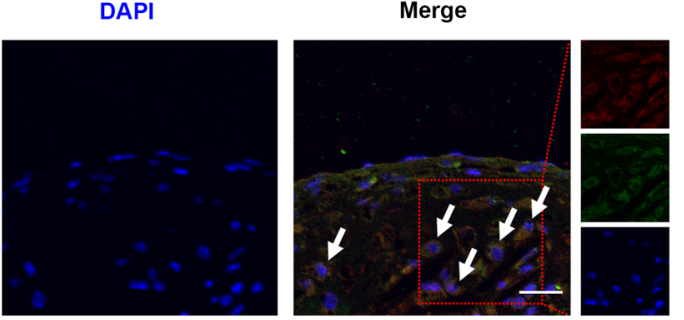

B
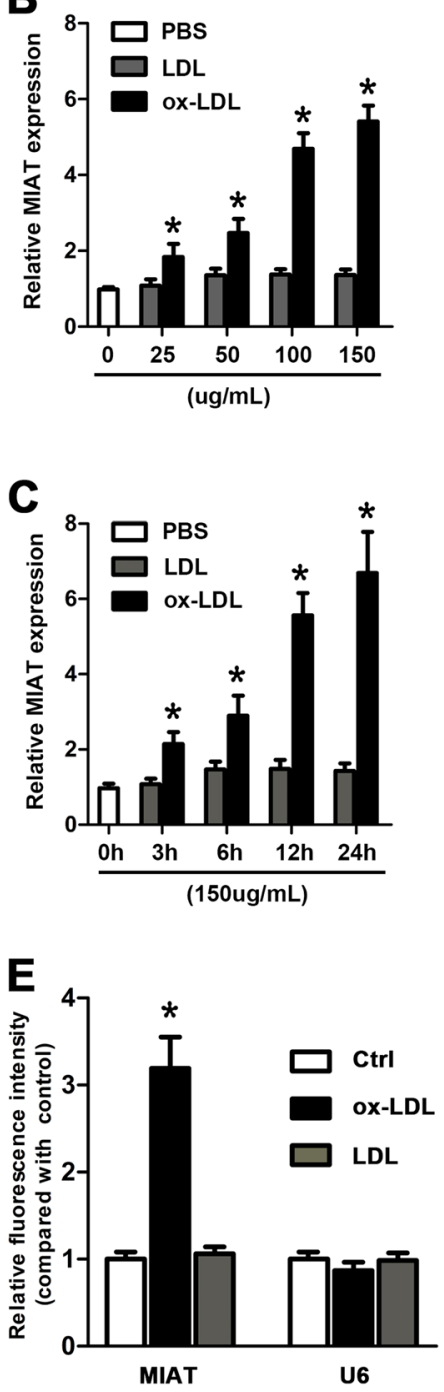

Fig. 2 Expression of MIAT expressed in macrophages in advanced atherosclerotic lesions and its upregulation in macrophages after oxLDL treatment. a RNA-fluorescent in situ hybridization (FISH) for MIAT (red) and immunostaining for Mac-3 (green) was performed on frozen aortic root sections from 16-week HFD-fed ApoE ${ }^{-/-}$mice. Arrows denote macrophages expressing MIAT. (Scale bar $=50 \mu m$ ). b, $\mathbf{c}$ qRT-PCR analysis of MIAT expression in ox-LDL or LDL-treated Raw264.7 cells at indicated dose $(0,25,50,100,150 \mu \mathrm{g} / \mathrm{mL})$ and at the indicated time $(0,3,6,12,24 \mathrm{~h})$ ( ${ }^{*}<<0.05$ oxLDL vs. LDL, $n=6$ /group). d, e RNA-FISH for MIAT (red) in Raw264.7 cells after ox-LDL treatment. Nuclei, blue. U6 was detected as a positive control. ( ${ }^{*} P<0.05$ oxLDL vs. Ctrl, $n=6 /$ group). Scale bar $=20 \mu \mathrm{m}$

the PBS-treated group (Supplementary Figure 3). However, we found a marked reduction of plaque formation in the aorta and aortic sinus in the MIAT shRNA-treated group compared with that of the scrambled RNA-treated group (Fig. 3d-g). These results suggested that MIAT knockdown could reduce the atherosclerotic burden in vivo independent of the plasma lipid profile and weight. 


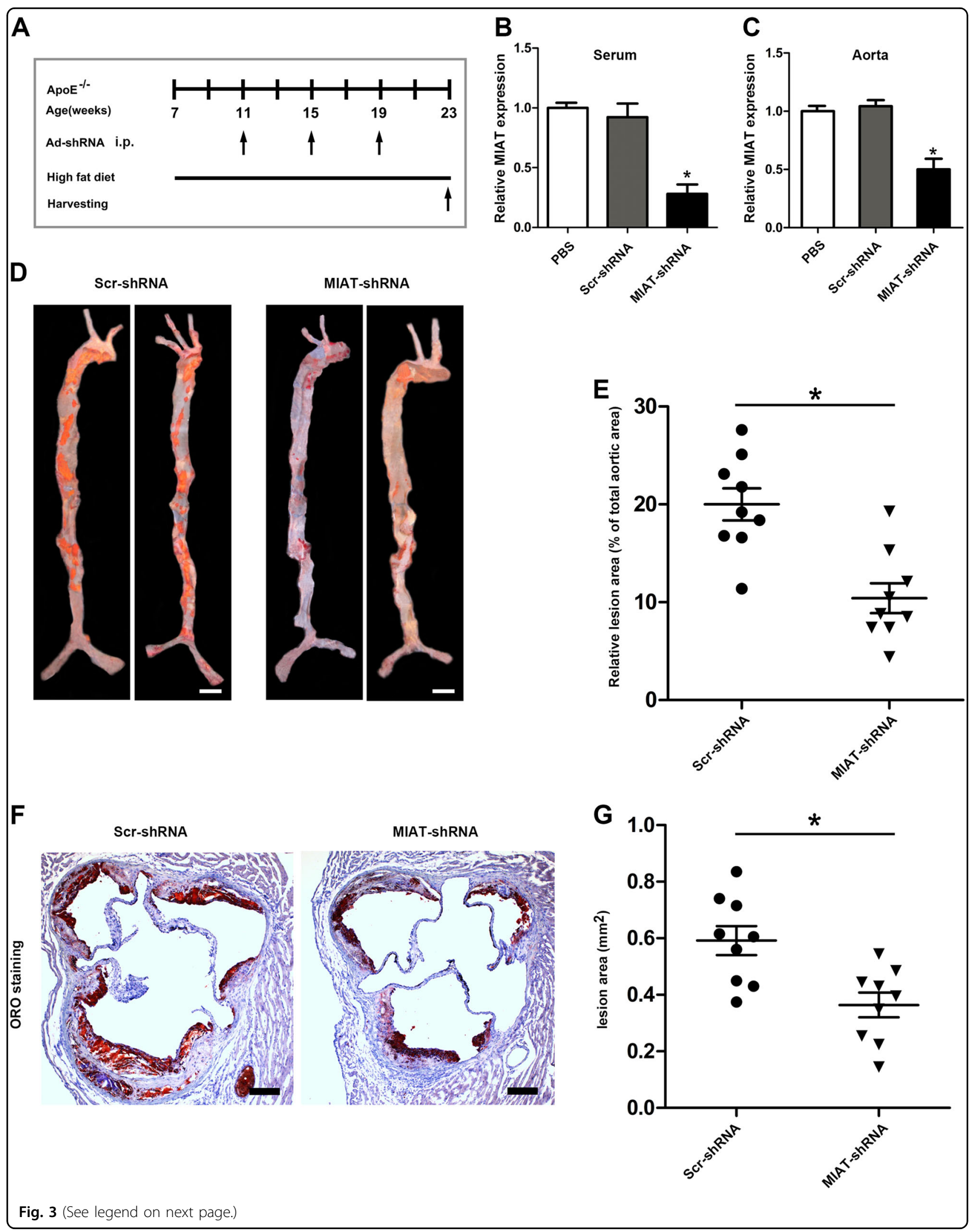


(see figure on previous page)

Fig. 3 Knockdown of MIAT attenuated atherosclerosis progression in $\mathrm{ApoE}^{-/-}$mice. a Flow charts showing the experimental protocol used in the in vivo studies. b, $\mathbf{c}$ qRT-PCR analysis of expression of MIAT in the serum and aorta of atherosclerotic lesions of PBS-treated, scrambled shRNA (ScrshRNA)- treated, and MIAT shRNA-treated ApoE ${ }^{-1}$ mice $\left({ }^{*} P<0.05\right.$, MIAT shRNA-treated mice vs. PBS-treated or Scr shRNA-treated mice, $n=6 /$ group). d, e ORO analysis of the percentage of lesion area to total aortic area in the Scr shRNA-treated and MIAT shRNA-treated ApoE ${ }^{-/-}$mice $\left({ }^{*} P<0.05\right.$, MIAT shRNA-treated mice vs. Scr shRNA-treated mice, $n=9$ /group). (Scale bar $=2 \mathrm{~mm}$ ). $\mathbf{f}, \mathbf{g}$ ORO analysis of the relative lesion area of aortic root area in the Scr shRNA-treated and MIAT shRNA-treated ApoE ${ }^{-1-}$ mice $\left({ }^{*} P<0.05\right.$, MIAT shRNA-treated mice vs. Scr shRNA-treated mice, $n=9 /$ group). (Scale $\operatorname{bar}=200 \mu \mathrm{m})$

\section{Knockdown of MIAT promoted atherosclerotic plaque stability in $\mathrm{ApoE}^{-1-}$ mice}

Atherosclerotic plaques in the MIAT shRNA-treated group contained smaller necrotic core areas compared with that of the scrambled RNA-treated group (Fig. 4a, b). Immunohistochemical analysis showed that the collagen content and the thickness of the fibrous cap were significantly increased in the atherosclerotic plaques of the MIAT shRNA-treated group, while the MOMA-2 ${ }^{+}$area was significantly decreased (Fig. $4 a-f$ ). In light of these changes, the plaque instability indexes in the MIAT shRNA-treated group were decreased by several magnitudes relative to the scrambled RNA-treated group (Fig. 4g). The plaque instability index was calculated according to the following formula: $\left[\left(\right.\right.$ oil red $\mathrm{O}^{+}$area $)+$ $\left(\right.$ MOMA- $^{+}$area $\left.)\right] /\left[\left(\alpha-\mathrm{SMA}^{+} \text {area }\right)+(\text { collagen area })\right]^{28}$. Both the decrease in necrotic area and increase in fibrous cap thickness indicated that the knockdown of MIAT promoted plaque stability.

\section{Knockdown of MIAT improved efferocytosis of atherosclerotic plaques in $\mathrm{ApoE}^{-/-}$mice}

A major cause of necrotic core formation is defective efferocytosis in advanced atherosclerotic plaques causing secondary necrosis of apoptosis cell-induced inflammation $^{18,19}$. We first evaluated intraplaque apoptotic cells by detecting TUNEL-positive nuclei (Fig. 5a, b) or caspase-3 activation (Fig. 5d, e) and observed fewer apoptotic cells in the lesions of the MIAT shRNAtreated group compared to those in the other groups. To further determine whether the reduced apoptotic cell number was associated with improved efferocytosis, we examined the number of 'free' apoptotic bodies (no costaining with macrophages) and 'not-free' apoptotic bodies (co-staining with macrophage marker Mac-3), which indicated an efferocytic event (Fig. 5a-d). Data are presented as the ratio of not-free/free apoptotic bodies, with higher values representing higher levels of efferocytosis. By either measurement, we determined that efferocytosis was significantly increased in the lesions of the MIAT shRNA-treated group compared with that of the scrambled RNA-treated group (Fig. 5c-f). These results suggested that improved efferocytosis from inhibiting MIAT may explain the decrease of necrotic core and the increase of plaque stability in the MIAT shRNA-treated group.

\section{Knockdown of MIAT promoted phagocytosis in vitro}

We next used an established in vitro phagocytosis assay to further elucidate the role of macrophage MIAT in efferocytosis (Fig. 6a). Previous studies suggested that oxLDL may play a pre-eminent function in atherosclerotic lesion formation. Therefore, we first used ox-LDL $(150 \mu \mathrm{g} / \mathrm{mL})$ in vitro to mimic the stimulation of macrophage apoptosis induction in atherosclerotic plaques (Supplementary Figure 4). We designed three MIAT small interfering RNAs (siRNAs) to assure the specificity of MIAT knockdown and that MIAT knockdown did not affect apoptosis in Raw264.7 cells (Supplementary Figure 5). Based on labeling with carboxy fluorescein succinimidyl ester (CFSE), we determined that MIAT knockdown markedly increased the uptake of Raw264.7 cells transfected with si-MIAT (Fig. 6b, c). We also found that MIAT knockdown markedly increased phagocytosis compared with that of the control groups, according to a pHrodo-based assay, which identified apoptotic cells within macrophage phagolysosomes (Fig. 6d, e). Consistently, the over-expression of MIAT markedly decreased the phagocytosis of apoptotic Raw264.7 cells compared with that of the control groups (Supplementary Figure 6). These in vitro results were consistent with the in vivo data, strongly suggesting that MIAT knockdown potently induced the clearance of diseased and apoptotic macrophages that had been exposed to oxidized phospholipids to simulate the atherosclerotic environment.

\section{MIAT upregulated anti-phagocytic molecule CD47 expression in a transcription-independent manner}

A recent study has shown that unengulfed macrophages express the anti-phagocytic molecule CD47 in advanced mouse and human atherosclerotic lesions ${ }^{24}$. We first found that the protein levels of CD47 were significantly upregulated after ox-LDL treatment in dose-dependent and time-dependent manners, which was consistent with MIAT expression after ox-LDL treatment (Fig. 7a, b). To determine whether CD47 might be directly responsible for the role of MIAT in defective efferocytosis, we performed in vitro gain-of-function and loss-of-function 


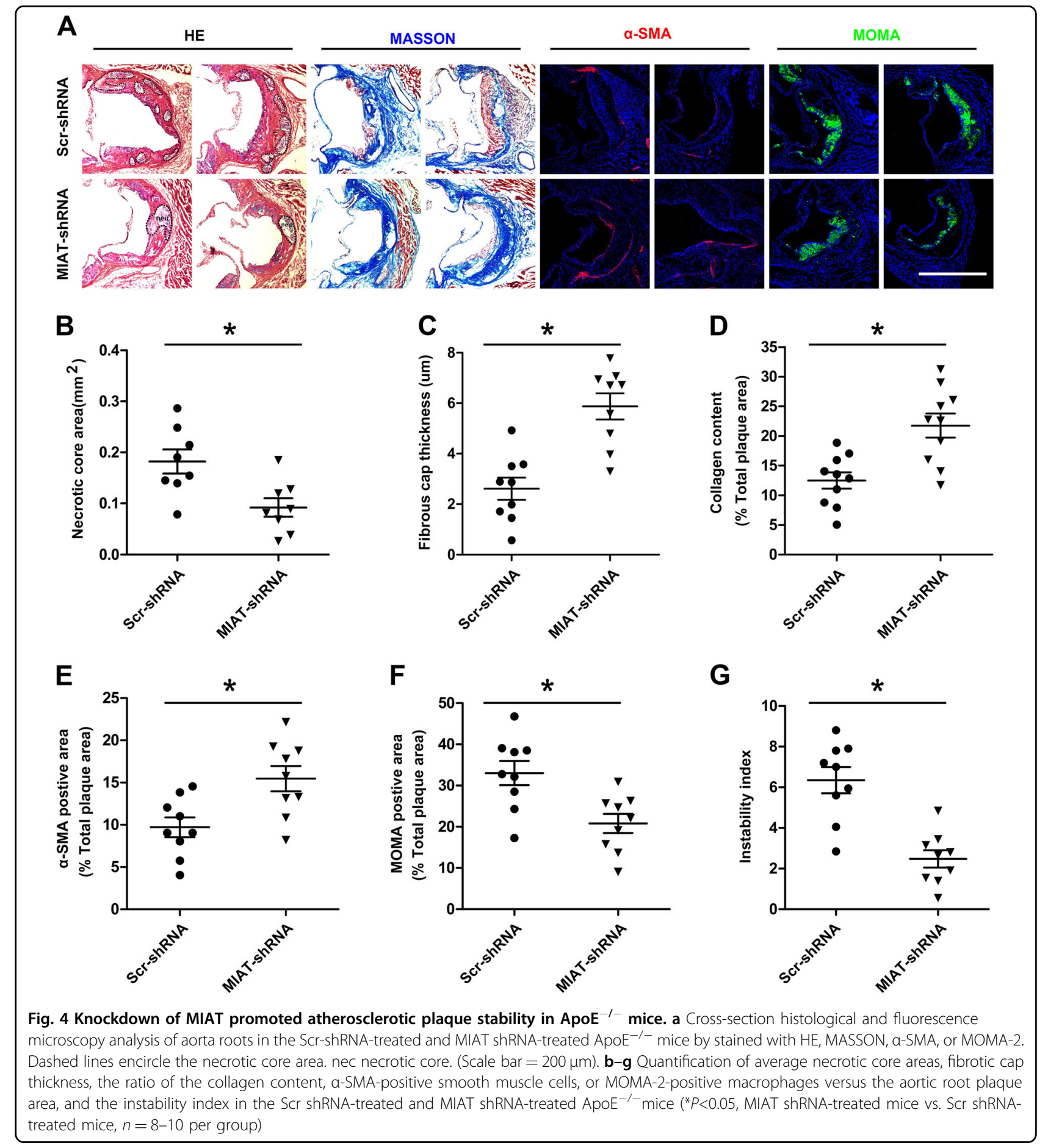

experiments in macrophages to investigate the effect of MIAT on CD47. Knockdown of MIAT using siRNA transfection decreased the protein levels of CD47 with oxLDL treatment compared with that of the control group (Fig. 7c). Conversely, the over-expression of MIAT by adenovirus infection efficiently increased ox-LDL-induced CD47 expression (Fig. 7d). We next investigated whether
MIAT upregulated CD47 expression in a transcriptiondependent manner. Interestingly, ox-LDL treatment did not affect the mRNA levels of CD47 in macrophages (Fig. 7e). Furthmore, downregulation and upregulation of MIAT did not change the CD47 mRNA levels (Fig. 7f). These data suggested that MIAT regulated CD47 expression in a transcription-independent manner. 


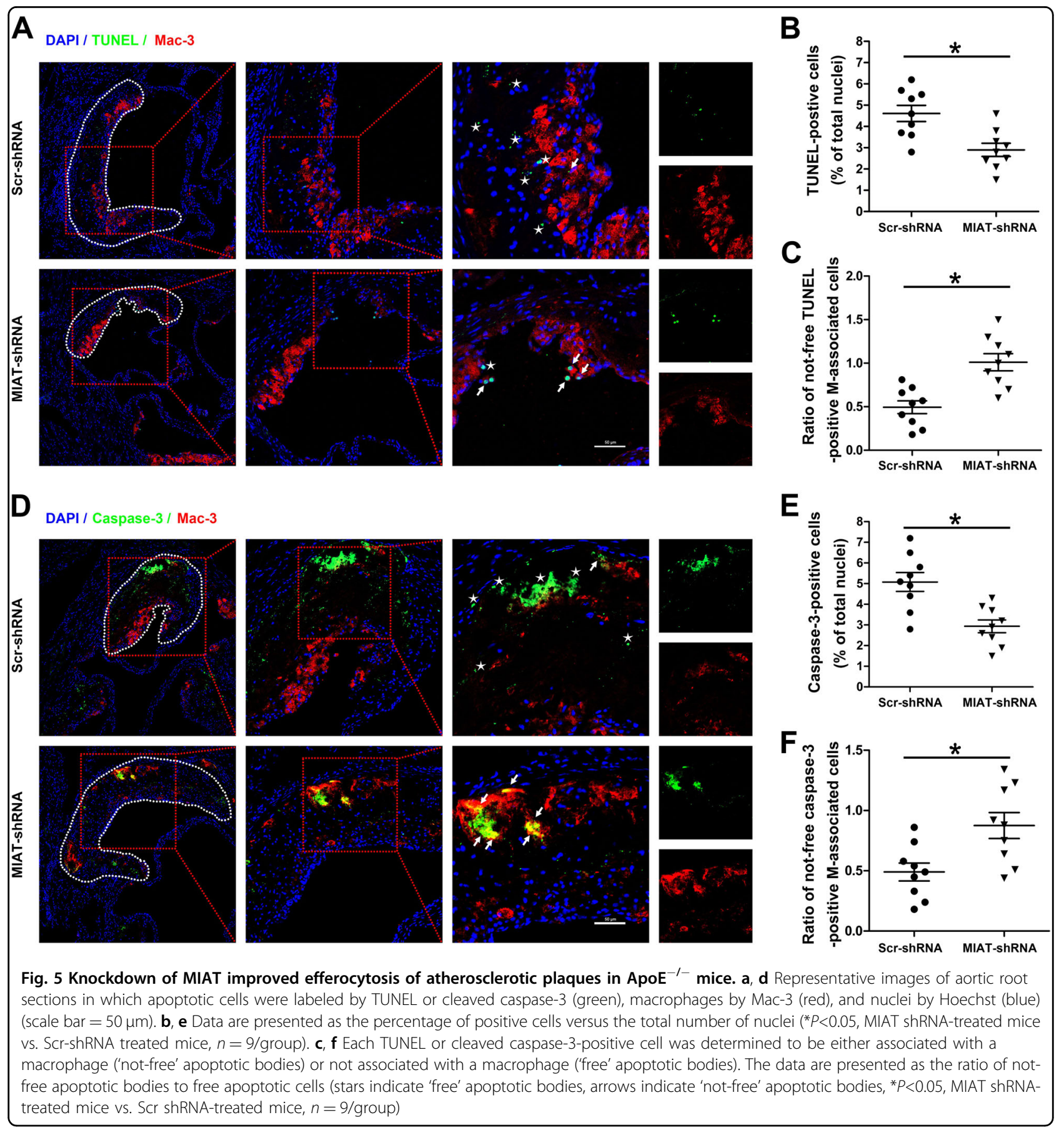

MIAT regulated CD47 expression by acting as a sponge of miR-149-5p

Numerous studies have shown that cytoplasmic lncRNAs can serve as natural miRNA sponges, which interferes with miRNA at the post-transcriptional level and decreases binding of endogenous miRNAs to target genes $^{5,29}$. We searched an online bioinformatics database (http://34.236.212.39/microrna/getGeneForm.do) for the potential miRNA recognition elements on MIAT. We found that the MIAT sequence contained eight putative
miR-149-5p sites in humans, and one putative miR-149$5 \mathrm{p}$ site in mice (Fig. 8a). Interestingly, using miRbase CD47 was also predicted to be a target gene of miR-149$5 \mathrm{p}$ (Fig. 8b). The sequences of MIAT or CD47 3'UTR along with the miR-149-5p binding site and the mutations MIAT mut or CD47 3'UTR mut were inserted downstream of the luciferase gene in the reporter plasmid for use in luciferase assays. The luciferase assay revealed that miR-149-5p transfection could reduce MIAT expression in the wild-type group compared with that in the negative 


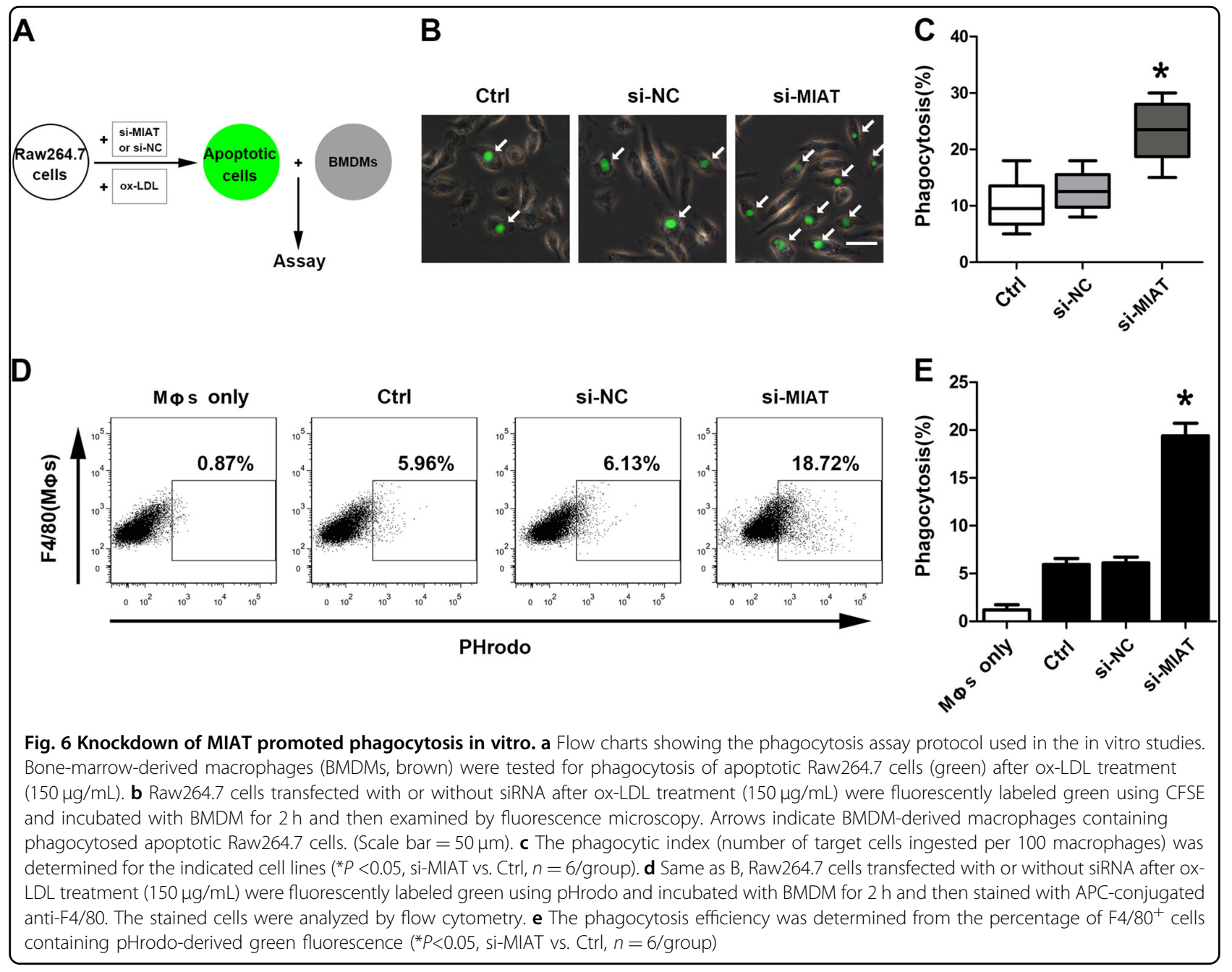

control group and this inhibition was reversed when the binding site was mutated, suggesting the same sequencespecific binding of miR-149-5p to MIAT or CD47 3'UTR (Fig. 8c, d).

To further determine the relationship among MIAT, miR-149-5p, and CD47, the CD47 3'UTR was cotransfected with miR-149-5p and the MIAT plasmid (pcDNA-MIAT) and analyzed by using luciferase assays. The results of the luciferase activity showed that MIAT could counteract the inhibitory effects of miR-149-5p on CD47 expression (Fig. 8e). Consistent with these findings, the over-expression of MIAT in Raw264.7 cells increased the protein levels of CD47 with this effect being counteracted by the over-expression of miR-149-5p (Fig. 8f). Knockdown of MIAT in Raw264.7 cells decreased the protein levels of $\mathrm{CD} 47$, which was also reversed by the down-regulation of miR-149-5p (Fig. 8g). These data suggested that MIAT modulated the expression of CD47 by interaction with miR-149-5p. Finally, to further confirm that MIAT and miR-149-5p were associated through a sponge, RNA immunoprecipitation was performed on
Raw264.7 cell extracts using an antibody specific for Ago2, a core component of the RNA-induced silencing complex (RISC). We found that higher MIAT and miR149-5p RNA levels were detected in the Ago2-containing miRNA ribonucleoprotein complexes (Fig. 8h). Therefore, these results indicated that MIAT acted as a miR-149-5p sponge and elevated CD47 expression.

\section{Discussion}

Recent studies have reported that IncRNA MIAT has critical functions in many cancers and microvascular dysfunction ${ }^{11,30}$, but little is known about its roles in the progression and instability of atherosclerosis. In the current study, we first found that MIAT expression levels in the serum were elevated in symptomatic patients with vulnerable plaques and $\mathrm{HFD}$-fed $\mathrm{ApoE}^{-1-}$ mice with advanced atherosclerosis. Expression of MIAT was primarily located in macrophages in the lesions and was also significantly increased after ox-LDL treatment. Furthermore, we determined that systemically delivered viral MIAT shRNA significantly reduced atherosclerosis 


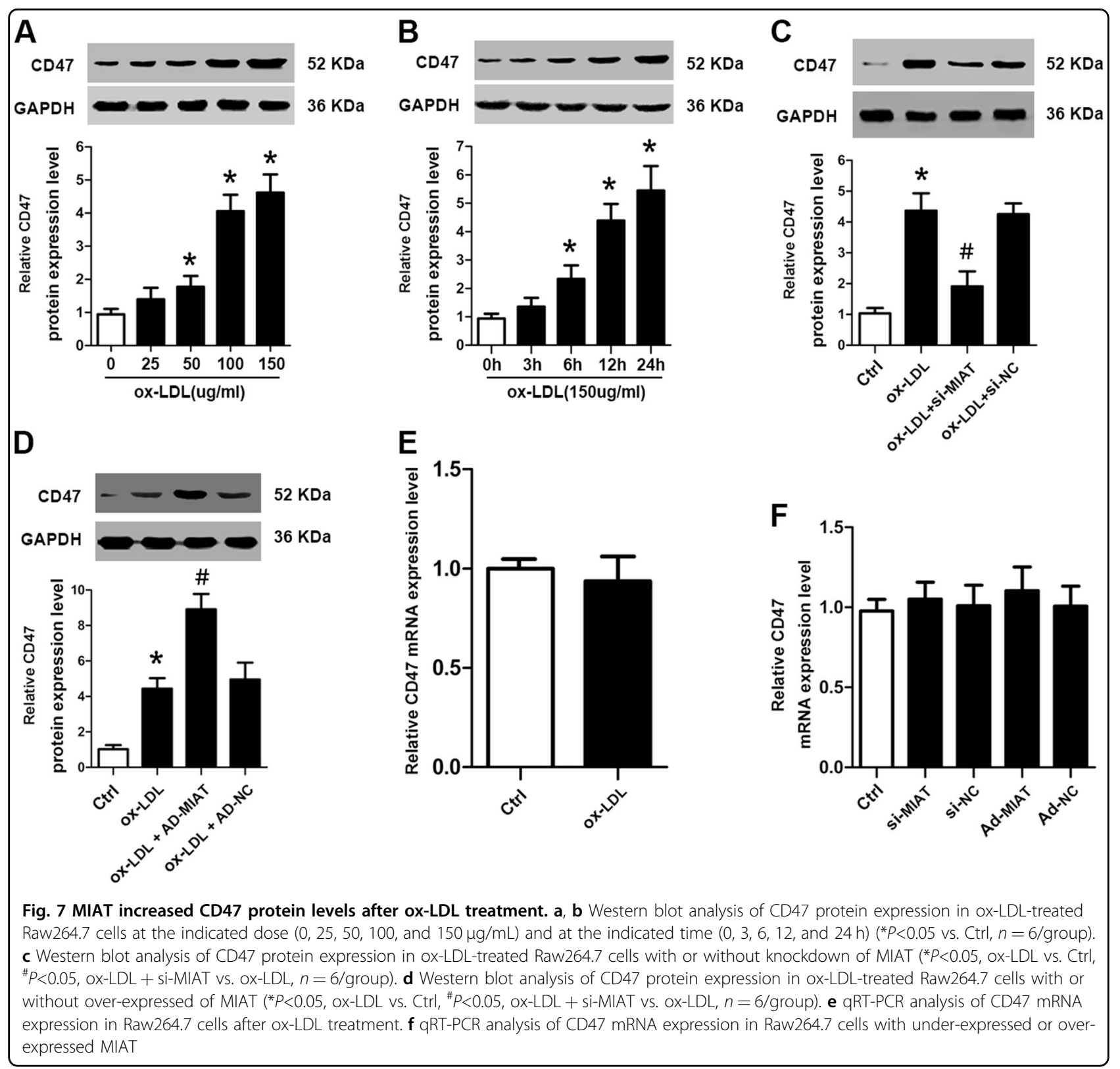

progression in vivo and promoted plaque stability by enhancing the clearance of apoptotic cells by lesion macrophages in $\mathrm{ApoE}^{-1-}$ mice. Specifically, our gain-offunction and loss-of-function experiments demonstrated that MIAT regulated miR-149-5p/CD47 axis to improve efferocytosis in vitro (summarized in Fig. 9). These findings support the rationale that MIAT acts as a novel biomarker and may serve as a therapeutic target for managing the progression and vulnerability of atherosclerotic disease.

LncRNAs are critical regulators of various biological functions and altered expression is closely associated with the development and progression of various diseases ${ }^{5,7,29}$. Recent studies have shown that several lncRNAs such as
lncRNA MeXis, lncRNA LeXis, and lncRNA SMILR are involved in macrophage cholesterol efflux, cholesterol metabolism, and SMCs proliferation, which are important in the process of atherosclerotic disease ${ }^{31-33}$. However, few studies have demonstrated regulatory functions of lncRNAs in atherosclerotic plaque vulnerability. Plaque vulnerability, which is responsible for plaque rupturing, is more highly correlated with plaque composition than with plaque size ${ }^{3,19,34}$. In the clinic, atherosclerotic plaque rupture is the most important mechanism of acute ischemic stroke $\mathrm{e}^{35,36}$. The average annual rate of ipsilateral stroke in patients with asymptomatic carotid stenosis is $\sim 0.5-1 \%$ per year and patients with low to moderate carotid stenosis also suffer strokes. Therefore, it is of great 


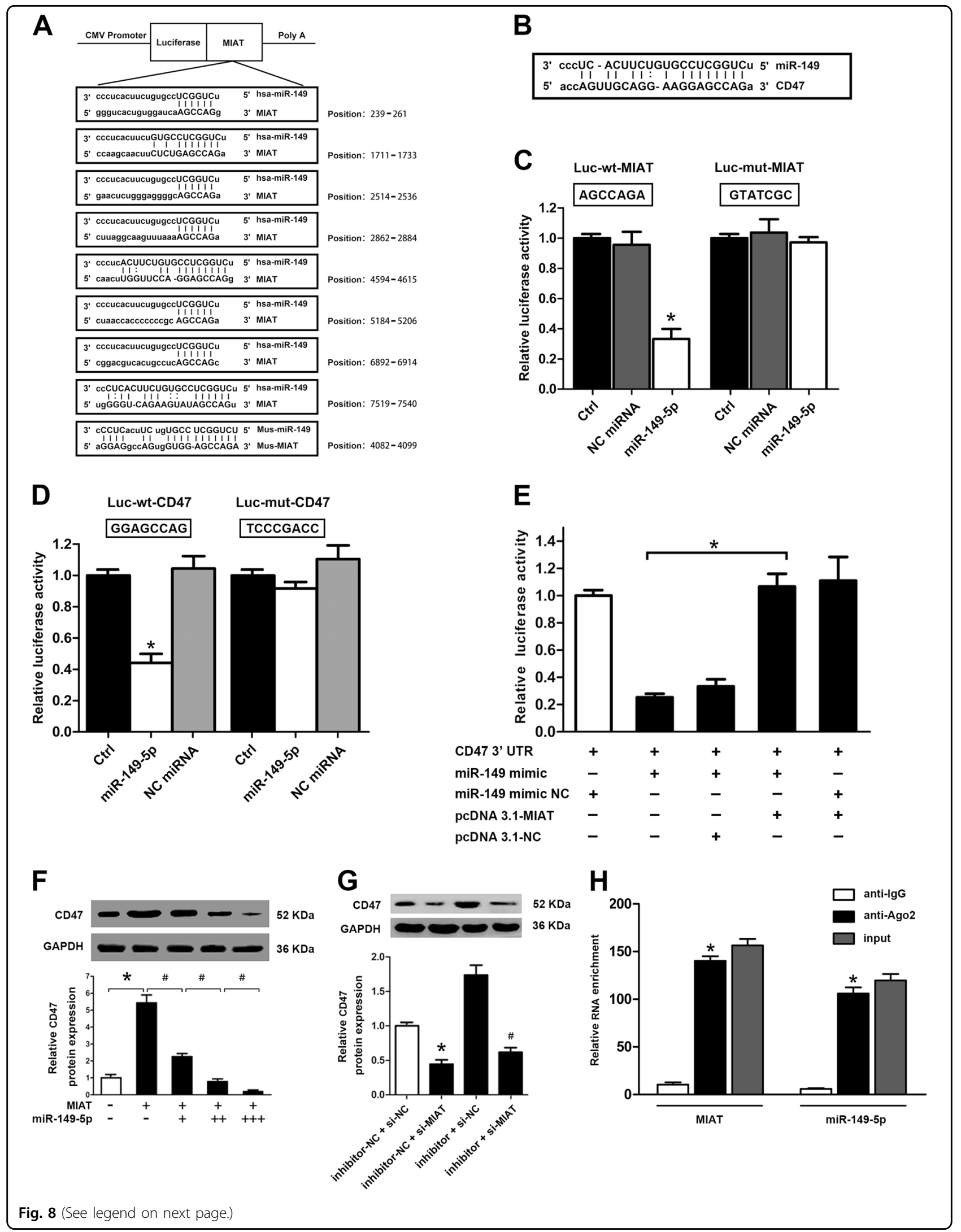


(see figure on previous page)

Fig. 8 MIAT regulated CD47 expression by acting as a sponge of miR-149-5p. a StarBase prediction indicated that the MIAT sequence contained eight putative miR-149-5p sites in humans and one putative miR-149-5p site in mice. b StarBase analysis indicated that CD47 was predicted to be a target gene of miR-149-5p. The positions of miR-149-5p binding sites on CD47 are shown. c The CDNA of MIAT was cloned downstream of the luciferase gene (Luc-MIAT-Wt) and transfected into HEK293 cells with miR-149-5p mimic or negative control (NC). To avoid unspecific binding, the miR-149-5p binding sites in MIAT were mutated to generate Luc-MIAT-Mut. Luciferase activity was detected $48 \mathrm{~h}$ after transfection ( ${ }^{*} P<0.05$ vs. NC, $n=6 /$ group). $\mathbf{d}$ Same as c, miR-149-5p mimics or NC were co-transfected with Luc-CD47-WT or MUT into HEK293 cells Luciferase activity was detected $48 \mathrm{~h}$ after transfection ( ${ }^{*} P<0.05$ vs. NC, $n=6 /$ group). e Luc-CD47-WT and miR-149-5p were co-transfected into HEK293 cells with MIAT plasmid or the vector to verify the competing endogenous RNA activity of MIAT. Luciferase activity was detected $48 \mathrm{~h}$ after transfection ${ }^{*} P<0.05$ vs. NC, $n=6$ /group). $\mathbf{f}$ Raw264.7 cells were transfected with different combinations of MIAT and miR-149-5p mimic and the cells were treated with ox-LDL. Western blot analysis was conducted to detect CD47 expression. The plus sign (+) corresponds to Ad-MIAT or $25 \mathrm{ng}$ of miR-149-5p mimic. The double plus signs $(++)$ corresponds to $50 \mathrm{ng}$ of miR-149-5p mimic. The triple plus signs $(+++)$ corresponds to $100 \mathrm{ng}$ of miR-149-5p mimic ( ${ }^{*} P<0.05$, Ad-MIAT vs. Ctrl, ${ }^{\#} P<0.05$, Ad-MIAT + miR-149-5p mimic vs. Ad-MIAT, $n=6 /$ group). g Raw264.7 cells with or without siMIAT were transfected with miR-149-5p inhibitor or inhibitor control and the cells were treated with ox-LDL. Western blot analysis was conducted to detect CD47 expression $\left({ }^{*} P<0.05\right.$, si-MIAT + inhibitor-NC vs. si-NC + inhibitor-NC, ${ }^{*} P<0.05$, si-MIAT + miR-149-5p inhibitor vs. si-MIAT + inhibitor NC, $n=6$ /group). $\mathbf{h}$ RNA-binding protein immunoprecipitation (RIP) assays were performed using input from cell lysates, normal mouse lgG, or antiAgo2. Relative expression levels of MIAT and miR-149-5p in Raw264.7 cells were detected by qRT-PCR analysis

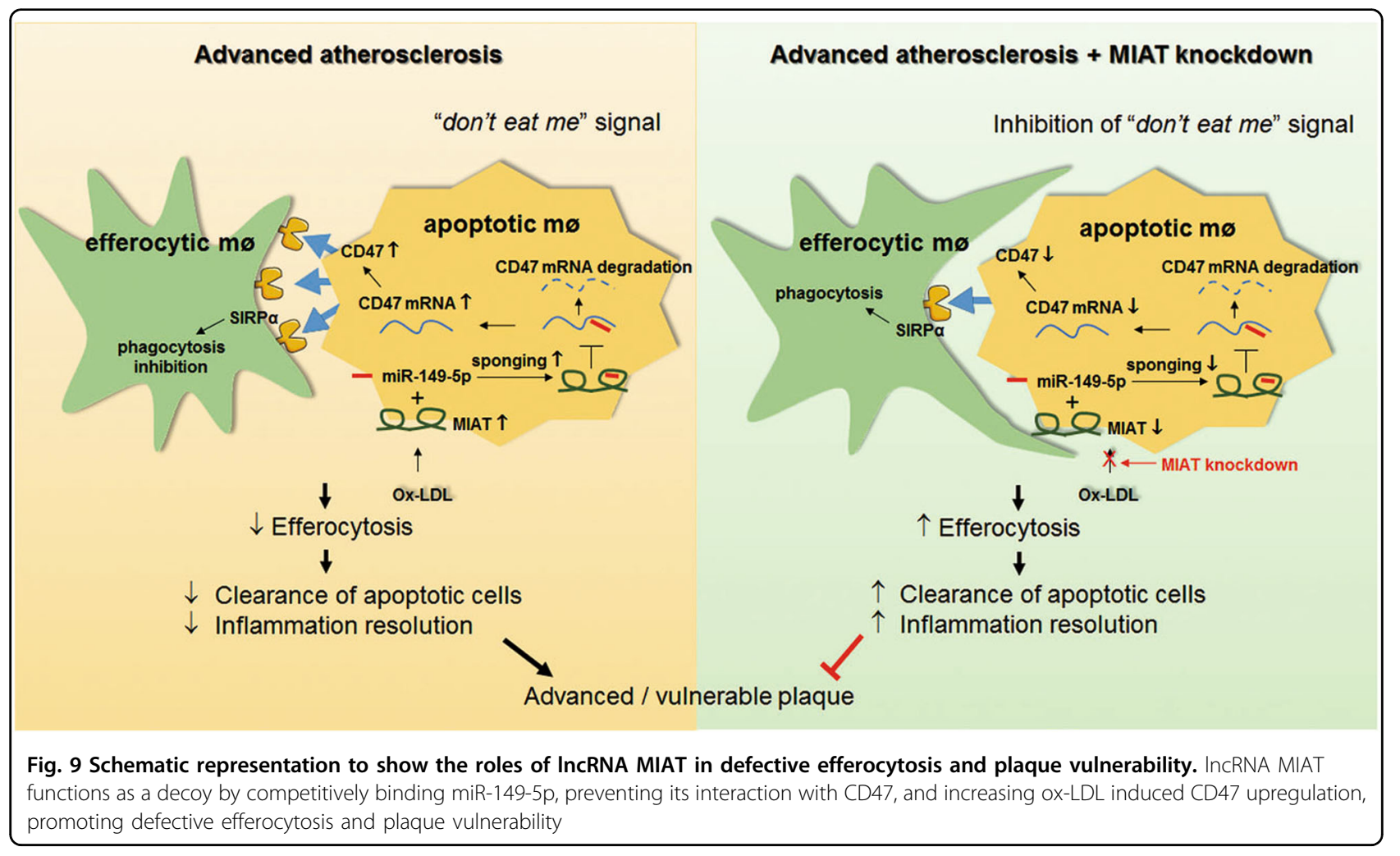

value to identify potential biomarkers of vulnerable plaques influencing the local disease process, which may provide reasonable solutions for these individuals.

In our current study, by comparing locally sampled serum of patients who were either asymptomatic or symptomatic (had recently suffered a transient ischemic attack or stroke), we identified MIAT as an important lncRNA being synthetized and released into the local cellular milieu. Consistent with these findings, recent studies have shown that MIAT is specifically up-regulated in the serum or plasma of diabetic cardiomyopathy patients and cataract patients ${ }^{37,38}$. In addition, a study reported that MIAT of peripheral blood leukocytes might be a potential diagnostic and prognostic indicator in ischemic stroke ${ }^{15}$. Furthermore, using RNA-FISH analysis we confirmed that MIAT was predominantly localized in the macrophages of advanced mouse atherosclerotic plaques. These results suggested that elevated MIAT positively correlated with atherosclerosis progression and instability. Due to the limitation in the number of clinical 
samples in our current study, a larger cohort study with follow-up information is needed, which would provide reliable data to fully determine whether MIAT is associated with prognosis or can serve as a new biomarker for vulnerable plaques.

Recent work has shown that MIAT knockdown alleviates diabetic-induced retinal neovascularization, vascular leakage, and inflammation in vivo ${ }^{11,37,39}$. To study the function of MIAT and examine the therapeutic potential in atherosclerosis, we knocked-down MIAT in vivo using systemically delivered viral MIAT shRNA in $\mathrm{ApoE}^{-1-}$ mice. Interestingly, MIAT knockdown significantly enhanced clearance of apoptotic cells by macrophages both in vivo and in vitro, which may explain the decrease of necrotic core and the increase of plaque stability. The anti-phagocytic molecule CD47 has been closely associated with the clearance of apoptotic cells and the formation of necrotic cores in plaque lesions ${ }^{24}$. In the present study, by performing in vitro gain-of-function and loss-of-function experiments in macrophages we determined that MIAT could positively regulate CD47 expression in a transcription-independent manner. Thus, it can be speculated from these results that CD47 may be a downstream target of MIAT in efferocytosis.

LncRNAs have been reported to be located in both the cytoplasm and nucleus of cells and subcellular localization patterns of lncRNAs provide new insight into their function and boost the hypotheses for potential molecular mechanisms ${ }^{5,7,40}$. First, by performing RNA FISH assays, we found that MIAT was localized in the cytoplasm of macrophages and levels in the cytoplasm and nucleus increased after ox-LDL treatment. The results indicated that MIAT might participate in post-transcriptional regulations in the cytoplasm ${ }^{41,42}$. Recently, a novel mechanism of post-transcriptional regulation by lncRNAs has been identified in which it functions as a natural miRNA sponge, interferes with miRNA pathways, and regulates the de-repression of miRNA targets ${ }^{41,43}$. Second, we predicted the interaction between MIAT and miR-149-5p using an online bioinformatics database and found that MIAT contains a target site of miR-149-5p. In addition, CD47 was predicted to be a target gene of miR-149-5p. Third, the over-expression of MIAT increased the level of CD47 and this effect was counteracted by the overexpression of miR-149-5p. Knockdown of MIAT decreased the level of $\mathrm{CD} 47$, which also was reversed by the down-regulation of miR-149-5p. Finally, we found that MIAT and miR-149-5p were in the same RISC complex. Therefore, we proposed that MIAT acted as a ceRNA to regulate CD47 expression by sponging miR149-5p in macrophages, which may be one of the mechanisms by which MIAT acts as a critical regulator in intraplaque efferocytosis and atherosclerotic plaque vulnerability.
The emergence of atherosclerosis-related lncRNAs as regulators of gene expression has greatly altered our understanding of the pathological mechanisms of atherosclerosis. In summary, we present evidence that lncRNA MIAT is a key regulatory factor for plaque vulnerability. These data also demonstrated how MIAT inhibited efferocytosis by targeting miR-149-5p/CD47 axis. The modulation of MIAT may provide an intriguing approach for prevention of atherosclerosis and tackling its treatment.

\section{Materials and methods \\ Ethics statement}

All the in vivo experiments complied with the Guide for Care and Use of Laboratory Animals published by the United States National Institutes of Health (NIH Publication, 8th Edition, 2011) and were approved by the Animal Care Committee of Tongji Medical College, Huazhong University of Science and Technology. The research protocol was approved by the ethical committee of Union Hospital, Huazhong University of Science and Technology, Wuhan, China. Written informed consent was obtained from each of the patients or the appropriate agents.

\section{Study population}

The study population consisted of 38 patients with carotid atherosclerosis and 20 control individuals. The participants were consecutively recruited from the Neurology Department and Health Examination Center between April 2016 and December 2017 at Union Hospital, Huazhong University of Science and Technology, Wuhan, China. The patients with ischemic stroke in the internal carotid artery underwent carotid vessel wall and brain magnetic resonance (MR) examinations within one week of symptoms onset. The exclusion criteria were as follows: (1) patients with evidence of cardioembolic stroke, (2) patients with hemorrhagic stroke, (3) history of radiation therapy of the neck, (4) other etiologies such as vasculitis or moyamoya disease, and (5) contraindications to magnetic resonance imaging (MRI).

\section{Magnetic resonance imaging and analysis}

For carotid vessel wall imaging, a previously published multi-contrast protocol was used for plaque detection that included T1-weighted, T2-weighted, 3D sampling perfection with application optimized contrasts using different flip angle evolutions (3D-SPACE), and postcontrast $\mathrm{T} 1$ and $\mathrm{T} 2$-weighted sequences ${ }^{44,45}$. Each lesion was classified according to the modified American Heart Association (AHA) classification for MRI ${ }^{46,47}$. Briefly, Type I-III were classified as stable plaques while types IV-V and VI were classified as unstable plaques. Each axial image was reviewed by two reviewers with consensus 
being reached. The reviewers were blinded to all clinical information.

\section{Plasma collection and storage}

Peripheral blood plasma from the patient group and control group were collected in ethylenediaminetetraacetic acid (EDTA) tubes and processed within $2 \mathrm{~h}$ by centrifugation at $1000 \times g$ at $4{ }^{\circ} \mathrm{C}$ for $10 \mathrm{~min}$. The plasma was gently transferred to RNase/DNase-free 2-mL EP tubes (Axygen, Union City, USA) and cryopreserved at $-80^{\circ} \mathrm{C}$ until use.

\section{Atherosclerosis animal model protocol}

A total of 100 seven-week-old male $\mathrm{ApoE}^{-1-}$ mice were purchased from Vital River Laboratory Animal Technology (Beijing, China). Thirty of the $\mathrm{ApoE}^{-/-}$mice were randomly divided into two groups, a normal chow diet group (NCD, $n=15$ ) and a high-fat diet group (HFD, $n=$ 15). The remaining $70 \mathrm{ApoE}^{-1-}$ mice were fed a high-fat diet and randomly divided into three groups, a PBS group $(n=10)$, Scr-shRNA group $(n=30)$, and MIAT-shRNA group $(n=30)$. The 7 -week-old male $\mathrm{ApoE}^{-1-}$ mice were fed a high-fat diet containing $21.2 \%$ fat (1.5\% cholesterol) and $16.7 \%$ protein for 16 weeks and the shRNA or PBS injections were then started at week 4 after initiation of the high-fat diet. The mice were treated intraperitoneally with shRNA adenoviral vector at a dose of $50 \mu \mathrm{l} / \mathrm{g}$ (virus titer: $1.0 \times 10^{13}$ viral genomes $/ \mathrm{mL}$ ) or PBS. The Scr shRNA-treated mice were used as a control group. The body weight of the mice was measured throughout the experimental period.

\section{Cell culture}

Raw264.7 cells and human embryonic kidney (HEK) $293 \mathrm{~T}$ cells were purchased from the Cell Resource Center of the Shanghai Institute for Biological Sciences (Shanghai, China). Both cell types were cultured in Dulbecco's modified Eagle's medium (DMEM; HyClone, USA) supplemented with $10 \%$ fetal bovine serum (FBS; Gibco, USA) and $1 \%$ penicillin/streptomycin (P/S; Thermo Fisher Scientific, Rockford, IL, USA) and were incubated in a humidified incubator at $37^{\circ} \mathrm{C}$ with $5 \% \mathrm{CO}_{2}$.

\section{RNA interference and virus infection}

RNA interference was performed as previously described $^{48}$. Negative control siRNA, and miR-149-5p miRNA mimics and inhibitors were designed and synthesized by Sangon Biotech (Shanghai, China). The adenovirus overexpressing lncRNA-MIAT was constructed by Shanghai R\&S Biotechnology Company (Shanghai, China).

\section{RNA extraction and quantitative real-time PCR}

Total RNA was isolated using Trizol (Invitrogen, USA). Real-time qRT-PCR was performed using a thermocycler
(Bio-Rad, Hercules, CA, USA) according to the manufacturer's recommendations. The primers for CD47 were 5'-CATGGCCCTCTTCTGATTTC-3' (forward) and 5'GGAGGTTGTATAGTCTTCTGATTGG-3' (reverse). The primers for LncRNA-MIAT were 5'-ATCCTCGA GACAAAGAGCCCTCTGCACTAG-3' (forward) and 5' ATCGGATCCGAGCAAATGGAGACAAAGGAC-3'

(reverse). The forward primer sequence for miR-149-5p was 5'-TCTGGCTCCGTGTCTTCACTCCC-3'. U6 and $\beta$-actin mRNA were used as housekeeping reference genes. The relative quantification of gene expression was performed using the comparative Ct method.

\section{Luciferase reporter assay}

The assays were performed as previously described ${ }^{48}$. In brief, for the luciferase reporter assay, the 3'UTR of MIAT or CD47 was amplified by PCR and inserted downstream of the firefly luciferase reporter gene in the reporter vector (RiboBio, China). Point mutations of the miR-149-5p targeting sites in the MIAT or CD47 $3^{\prime}$ UTR were generated. Luciferase activity was detected using the DualLuciferase Reporter Assay System (Promega, Madison, WI, USA).

\section{RNA-binding protein immunoprecipitation (RIP) assay}

RIP assays were performed using the EZ-Magna RIP Kit (Millipore, USA). Briefly, cells were collected and lysed in complete RIP lysis buffer. The cell extracts were then incubated with RIP buffer containing magnetic beads conjugated to anti-Ago2 antibody (Millipore, USA). Samples were incubated with proteinase K with shaking to digest the proteins and the immunoprecipitated RNA was isolated. MIAT and miR-149-5p levels in the precipitates were determined by qRT-PCR analysis.

\section{In vitro phagocytosis assays}

For the pHrodo and flow cytometry-based assays bonemarrow-derived macrophages (BMDMs) were prepared for phagocytosis ${ }^{49,50}$. The BMDMs $\left(5 \times 10^{4}\right)$ were seeded overnight in 24-well culture plates. Raw264.7 cells were induced as apoptotic cells using ox-LDL $(150 \mu \mathrm{g} / \mathrm{mL})$ for $24 \mathrm{~h}$ and then labeled with $100 \mathrm{ng} \mathrm{mL}^{-1}$ of pHrodo Green AM Intracellular $\mathrm{pH}$ Indicator (Thermo Fisher Scientific, USA) according to the manufacturer's instructions. After incubating the BMDMs in serum-free medium for $2 \mathrm{~h}$, $2 \times 10^{5}$ pHrodo-labeled Raw264.7 cells were added to the BMDMs. After incubation for $2 \mathrm{~h}$ at $37^{\circ} \mathrm{C}$, the BMDMs were washed extensively, then stained with alkaline phosphatase-conjugated (APC) anti-F4/80 antibody and were analyzed by flow cytometry. The pHrodo dyes do not fluoresce under neutral $\mathrm{pH}$ but become fluorescent in acidic environments, such as phagolysosome. The efficiency of phagocytosis was determined from the percentage of $\mathrm{F} 4 / 80^{+}$cells containing pHrodo-derived green 
fluorescence. For the microscopy-based assay, apoptotic Raw264.7 cells were used for the preparation and incubation of BMDMs as detailed for the flow cytometrybased assay ${ }^{50,51}$. Meanwhile, the apoptotic Raw264.7 cells were labeled with $2.5 \mu \mathrm{M}$ carboxyfluorescein succinimidyl ester (CFSE) using a CFSE Cell Proliferation Kit (C34554; Life Technologies, Burlington, Ontario, Canada). After $2 \mathrm{~h}$ incubation at $37^{\circ} \mathrm{C}$, the BMDMs were extensively washed and imaged using an inverted microscope (Carl Zeiss Axiovert S100 TV). The number of BMDMs containing CFSE + apoptosis cells per 100 BMDMs was calculated and the phagocytosis efficiency accordingly evaluated.

\section{Biochemical parameters}

As previously described ${ }^{28,48}$, total cholesterol (TC), triglycerides (TG), low-density lipoprotein cholesterol (LDL-c), and high-density lipoprotein cholesterol (HDLc) were enzymatically measured (Wako Chemicals, USA) following the manufacturer's protocols.

\section{Histology and immunostaining}

Serial sections $(6 \mu \mathrm{m}$ thick) of the aortic root (3-5 sections per mouse) were stained with hematoxylin and eosin (HE), Oil Red O (ORO), or Masson's trichrome (MASSON) and the microscopic images were then collected. Quantitative immunostaining was performed using primary antibodies for capase-3 (1:200, Cell Signaling Technology [CST], Danvers, MA, USA), Mac-3 (1:200, Abcam, USA), MOMA-2 (1:200, Abcam, USA), or $\alpha-$ smooth muscle actin ( $\alpha$-SMA, 1:100, Abcam, USA). Fluorescently labeled secondary antibodies were used for detection. The quantification of colocalized signals and the percentage of positive area in the images were performed using NIS Elements AR Imaging Software 4.10 (Nikon) or ImageJ 1.41 software (Image Processing and Analysis in Java; National Institutes of Health, Bethesda, MD, USA).

\section{In situ detection of apoptotic cells}

Apoptotic cells in aortic root cryo-sections were determined using an In-Situ Apoptosis Fluorescein Detection Kit (Roche, Sigma, USA) according to the manufacturer's instructions.

\section{RNA fluorescent in situ hybridization}

Cy3-labeled IncRNA-MIAT and DAPI-labeled U6 probes were obtained from RiboBio (Guangzhou, China). RNA-FISH assays were carried out using a fluorescent in situ hybridization kit (RiboBio, Guangzhou, China) following the manufacturer's instructions.

\section{Western blot analysis}

Proteins were extracted from cell lysates using RIPA buffer containing protease inhibitors. Eighty $\mu \mathrm{g}$ protein was separated by $6 \%$ sodium dodecyl sulfate-polyacrylamide gel electrophoresis (SDS-PAGE) and transferred to polyvinylidene difluoride (PVDF) membrane. The membranes were incubated overnight with antibodies to CD47 (1:400, Abcam, USA) and capase-3 (1:500, CST, USA) followed by incubation with the corresponding secondary antibody. The bands were visualized using the enhanced chemiluminescence method. Protein expression was quantified and normalized to GAPDH (1:1000, Proteintech, China).

\section{Statistics}

The data represent the mean \pm standard error of the mean (SEM). A Kolmogorov-Smirnov normality test was performed to determine whether the data showed normal distribution. Comparisons between the controls and treatment groups were performed using either a Student's $t$-test or one-way ANOVA. The statistical significance is shown as described in the figure legends. The Mann-Whitney $U$ test or Student's $t$-test was used for continuous variables between patients and controls. Statistical significance was defined as $P<0.05$ for all tests. Statistical analyses were performed using the Statistical Package for the Social Sciences (SPSS) version 17.0 software (SPSS Inc., Chicago, IL, USA) and GraphPad Prism 6.

\section{Acknowledgements \\ This work was supported by National Key Research and Development Program of China (No. 2018YFC1312200), National Natural Science Foundation of China (No. 81571119 to B.H., No.81771249 to Y.X., No. 81860222 to C.Q., No. 81801172 to S.C., No. 81601027 to Y.L.), Major refractory diseases pilot project of clinical collaboration with Chinese \& Western Medicine (SATCM-20180339).}

\section{Author contributions}

Z.-Y. and S.Y. performed the experiments; Z.-Y., S.Y., and Y.-X. analyzed the data and wrote the manuscript; R.-H., S.C., B.-L., X.-L., S.-C., H.J., Y.L., and L.M. edited the manuscript; B.H. and C.Q. designed the research.

\section{Conflict of interest}

The authors declare that they have no conflict of interest.

\section{Publisher's note \\ Springer Nature remains neutral with regard to jurisdictional claims in} published maps and institutional affiliations.

Supplementary Information accompanies this paper at (https://doi.org/ 10.1038/s41419-019-1409-4).

Received: 21 October 2018 Revised: 18 January 2019 Accepted: 29 January 2019

Published online: 12 February 2019

\footnotetext{
References

1. Fowkes, F. G. et al. Comparison of global estimates of prevalence and risk factors for peripheral artery disease in 2000 and 2010: a systematic review and analysis. Lancet 382, 1329-1340 (2013).

2. Ridker, P. M. et al. Antiinflammatory therapy with canakinumab for atherosclerotic disease. N. Engl. J. Med. 377, 1119-1131 (2017).
} 
3. Toutouzas, K. et al. Vulnerable plaque imaging: updates on new pathobiological mechanisms. Eur. Heart J. 36, 3147-3154 (2015).

4. Lerman, J. B. et al. Coronary plaque characterization in psoriasis reveals highrisk features that improve after treatment in a prospective observational study. Circulation 136, 263-276 (2017).

5. Kopp, F. \& Mendell, J. T. Functional classification and experimental dissection of long noncoding RNAs. Cell 172, 393-407 (2018).

6. Kumar, S., Williams, D., Sur, S., Wang, J. Y. \& Jo, H. Role of flow-sensitive microRNAs and long noncoding RNAs and in vascular dysfunction and atherosclerosis. Vascul. Pharmacol. https:/doi.org/10.1016/j.vph.2018.10.001 (2018).

7. Sallam, T., Sandhu, J. \& Tontonoz, P. Long noncoding RNA discovery in cardiovascular disease: decoding form to function. Circ. Res. 122, 155-166 (2018).

8. Bayoumi, A. S., Aonuma, T., Teoh, J. P., Tang, Y. L. \& Kim, I. M. Circular noncoding RNAs as potential therapies and circulating biomarkers for cardiovascular diseases. Acta Pharmacol. Sin. 39, 1100-1109 (2018).

9. Lucas, T., Bonauer, A. \& Dimmeler, S. RNA therapeutics in cardiovascular disease. Circ. Res. 123, 205-220 (2018)

10. Barry, G. et al. The long non-coding RNA Gomafu is acutely regulated in response to neuronal activation and involved in schizophrenia-associated alternative splicing. Mol. Psychiatry 19, 486-494 (2014).

11. Yan, B. et al. IncRNA-MIAT regulates microvascular dysfunction by functioning as a competing endogenous RNA. Circ. Res. 116, 1143-1156 (2015).

12. Vausort, M., Wagner, D. R. \& DevauX, Y. Long noncoding RNAs in patients with acute myocardial infarction. Circ. Res. 115, 668-677 (2014).

13. Sun, C. et al. Long non-coding RNA MIAT in development and disease: a new player in an old game. J. Biomed. Sci. 25, 23 (2018).

14. Arslan, S. et al. Long non-coding RNAs in the atherosclerotic plaque. Atherosclerosis 266, 176-181 (2017)

15. Zhu, M. et al. Peripheral blood leukocyte expression of IncRNA MIAT and its diagnostic and prognostic value in ischemic stroke. J. Stroke Cerebrovasc. Dis. 27, 326-337 (2018).

16. Koelwyn, G. J., Corr, E. M., Erbay, E. \& Moore, K. J. Regulation of macrophage immunometabolism in atherosclerosis. Nat. Immunol. 19, 526-537 (2018).

17. Tabas, I. \& Lichtman, A. H. Monocyte-macrophages and T cells in atherosclerosis. Immunity 47, 621-634 (2017).

18. Kasikara, C., Doran, A. C., Cai, B. \& Tabas, I. The role of non-resolving inflammation in atherosclerosis. J. Clin. Invest. 128, 2713-2723 (2018).

19. Silvestre-Roig, C. et al. Atherosclerotic plaque destabilization: mechanisms, models, and therapeutic strategies. Circ. Res. 114, 214-226 (2014).

20. Henson, P. M. Cell removal: efferocytosis. Annu. Rev. Cell. Dev. Biol. 33, 127-144 (2017).

21. Proto, J. D. et al. Regulatory T Cells Promote Macrophage Efferocytosis during Inflammation Resolution. Immunity, https://doi.org/10.1016/j. immuni.2018.07.015 (2018).

22. Doran, A. C. et al. CAMKllgamma suppresses an efferocytosis pathway in macrophages and promotes atherosclerotic plaque necrosis. J. Clin. Invest. 127, 4075-4089 (2017).

23. Seneviratne, A. N. et al. Interferon regulatory factor 5 controls necrotic core formation in atherosclerotic lesions by impairing efferocytosis. Circulation 136 1140-1154 (2017).

24. Kojima, Y. et al. CD47-blocking antibodies restore phagocytosis and prevent atherosclerosis. Nature 536, 86-90 (2016).

25. Matlung, H. L., Szilagyi, K., Barclay, N. A. \& van den Berg, T. K. The CD47SIRPalpha signaling axis as an innate immune checkpoint in cancer. Immunol. Rev. 276, 145-164 (2017).

26. Weiskopf, K. Cancer immunotherapy targeting the CD47/SIRPa axis. Eur. J. Cancer 76, 100-109 (2017).

27. Chan, A. C. \& Carter, P. J. Therapeutic antibodies for autoimmunity and inflammation. Nat. Rev. Immunol. 10, 301-316 (2010).
28. Dong, M. et al. Cold exposure promotes atherosclerotic plaque growth and instability via UCP1-dependent lipolysis. Cell Metab. 18, 118-129 (2013).

29. Sullenger, B. A. \& Nair, S. From the RNA world to the clinic. Science $\mathbf{3 5 2}$, 1417-1420 (2016)

30. Mitra, R. et al. Decoding critical long non-coding RNA in ovarian cancer epithelial-to-mesenchymal transition. Nat. Commun. 8, 1604 (2017).

31. Sallam, T. et al. Transcriptional regulation of macrophage cholesterol efflux and atherogenesis by a long noncoding RNA. Nat. Med. 24, 304-312 (2018).

32. Sallam, T. et al. Feedback modulation of cholesterol metabolism by the lipidresponsive non-coding RNA LeXis. Nature 534, 124-128 (2016).

33. Ballantyne, M. D. et al. Smooth muscle enriched long noncoding RNA (SMILR) regulates cell proliferation. Circulation 133, 2050-2065 (2016).

34. Benagiano, $M$. et al. $\beta 2$ glycoprotein I recognition drives th1 inflammation in atherosclerotic plaques of patients with primary antiphospholipid syndrome. J. immunol. 198, 2640-2648 (2017)

35. Boehme, A. K. Esenwa, C. \& Elkind, M. S. Stroke risk factors, genetics, and prevention. Circ. Res. 120, 472-495 (2017).

36. Wendelboe, A. M. \& Raskob, G. E. Global burden of thrombosis: epidemiologic aspects. Circ. Res. 118, 1340-1347 (2016).

37. Zhou, $X$. et al. IncRNA MIAT functions as a competing endogenous RNA to upregulate DAPK2 by sponging miR-22-3p in diabetic cardiomyopathy. Cell Death Dis. 8, e2929 (2017).

38. Shen, Y. et al. Role of long non-coding RNA MIAT in proliferation, apoptosis and migration of lens epithelial cells: a clinical and in vitro study. J. Cell. Mol. Med. 20, 537-548 (2016).

39. Jiang, Q. et al. Long non-coding RNA-MIAT promotes neurovascular remodeling in the eye and brain. Oncotarget 7, 49688-49698 (2016).

40. Adams, B. D., Parsons, C., Walker, L., Zhang, W. C. \& Slack, F. J. Targeting noncoding RNAs in disease. J. Clin. Invest. 127, 761-771 (2017).

41. Tay, Y., Rinn, J. \& Pandolfi, P. P. The multilayered complexity of ceRNA crosstalk and competition. Nature 505, 344-352 (2014).

42. Thomson, D. W. \& Dinger, M. E. Endogenous microRNA sponges: evidence and controversy. Nat. Rev. Genet. 17, 272-283 (2016).

43. Cesana, M. et al. A long noncoding RNA controls muscle differentiation by functioning as a competing endogenous RNA. Cell 147, 358-369 (2011).

44. Sun, B. et al. Association between carotid plaque characteristics and acute cerebral infarction determined by MRI in patients with type 2 diabetes mellitus. Cardiovasc. Diabetol. 16, 111 (2017).

45. Zhao, X. et al. Prevalence and characteristics of carotid artery high-risk atherosclerotic plaques in chinese patients with cerebrovascular symptoms: a chinese atherosclerosis risk evaluation II study. J. Am. Heart Assoc. 6, https://doi. org/10.1161/jaha.117.005831 (2017).

46. Cai, J. M. et al. Classification of human carotid atherosclerotic lesions with in vivo multicontrast magnetic resonance imaging. Circulation 106, 1368-1373 (2002).

47. Saam, T. et al. Expansive arterial remodeling of the carotid arteries and its effect on atherosclerotic plaque composition and vulnerability: an in-vivo black-blood 3T CMR study in symptomatic stroke patients. J. Cardiovasc. Magn. Reson. 18, 11 (2016).

48. Yang, S. et al. MicroRNA-23a-5p promotes atherosclerotic plaque progression and vulnerability by repressing ATP-binding cassette transporter A1/G1 in macrophages. J. Mol. Cell. Cardiol. 123, 139-149 (2018).

49. Takahashi, D. et al. Flow cytometric quantitation of platelet phagocytosis by monocytes using a pH-sensitive dye, pHrodo-SE. J. Immunol. Methods 447, 57-64 (2017).

50. Chen, J. et al. SLAMF7 is critical for phagocytosis of haematopoietic tumour cells via Mac-1 integrin. Nature 544, 493-497 (2017).

51. Crauwels, P. et al. Apoptotic-like Leishmania exploit the host's autophagy machinery to reduce T-cell-mediated parasite elimination. Autophagy 11, 285-297 (2015). 